\title{
Characterization and Comparative Analysis of the Complete Chloroplast Genome of the Critically Endangered Species Streptocarpus teitensis (Gesneriaceae)
}

\author{
Cornelius M. Kyalo ${ }^{1}$, , ${ }^{1,2,3}$ Andrew W. Gichira, ${ }^{1,2,3}$ Zhi-Zhong Li, ${ }^{1,2,3}$ Josphat K. Saina, ,2,3 \\ Itambo Malombe, ${ }^{4}$ Guang-Wan $H u\left(\mathbb{D},{ }^{1,3}\right.$ and Qing-Feng Wang $\mathbb{D}^{1,3}$ \\ ${ }^{1}$ Key Laboratory of Aquatic Botany and Watershed Ecology, Wuhan Botanical Garden, Chinese Academy of Sciences, \\ Wuhan 430074, China \\ ${ }^{2}$ University of Chinese Academy of Sciences, Beijing 100049, China \\ ${ }^{3}$ Sino-Africa Joint Research Center, Chinese Academy of Sciences, Wuhan 430074, China \\ ${ }^{4}$ East African Herbarium, National Museums of Kenya, P.O. Box 45166-00100, Nairobi, Kenya
}

Correspondence should be addressed to Guang-Wan Hu; guangwanhu@wbgcas.cn and Qing-Feng Wang; qfwang@wbgcas.cn

Received 18 October 2017; Revised 27 December 2017; Accepted 28 January 2018; Published 25 March 2018

Academic Editor: Stanley Brul

Copyright ( 2018 Cornelius M. Kyalo et al. This is an open access article distributed under the Creative Commons Attribution License, which permits unrestricted use, distribution, and reproduction in any medium, provided the original work is properly cited.

Streptocarpus teitensis (Gesneriaceae) is an endemic species listed as critically endangered in the International Union for Conservation of Nature (IUCN) red list of threatened species. However, the sequence and genome information of this species remains to be limited. In this article, we present the complete chloroplast genome structure of Streptocarpus teitensis and its evolution inferred through comparative studies with other related species. S. teitensis displayed a chloroplast genome size of $153,207 \mathrm{bp}$, sheltering a pair of inverted repeats (IR) of 25,402 bp each split by small and large single-copy (SSC and LSC) regions of 18,300 and 84,103 bp, respectively. The chloroplast genome was observed to contain 116 unique genes, of which 80 are proteincoding, 32 are transfer RNAs, and four are ribosomal RNAs. In addition, a total of 196 SSR markers were detected in the chloroplast genome of Streptocarpus teitensis with mononucleotides (57.1\%) being the majority, followed by trinucleotides (33.2\%) and dinucleotides and tetranucleotides (both $4.1 \%$ ), and pentanucleotides being the least (1.5\%). Genome alignment indicated that this genome was comparable to other sequenced members of order Lamiales. The phylogenetic analysis suggested that Streptocarpus teitensis is closely related to Lysionotus pauciflorus and Dorcoceras hygrometricum.

\section{Introduction}

Streptocarpus Lindley (Cape primroses) are herbaceous plant species of the family Gesneriaceae and exhibit either annual or perennial lifeforms. The genus hosts approximately 176 species [1], distributed in Madagascar, Comoros islands, and some regions of Africa [2,3], with the regions sharing no single species [4]. The genus, like most members of the family Gesneriaceae, hosts some species of great horticultural importance such as Streptocarpus ionanthus which contribute a large percentage to the world's flower industry [5].
Streptocarpus teitensis [6], previously known as Saintpaulia teitensis, is an endemic of Taita hills, Kenya, the northernmost stretch of the Eastern arc mountain forests. The species has experienced a drastic reduction in the distribution range over the past, and it was recorded to be restricted to a single site $\left(<2.5 \mathrm{~km}^{2}\right)$ in the wild, Mbololo Hill [7], although more than half of the range has deteriorated of late (personal observation, 2017). This restriction in distribution coupled with other threats facing this species has led to its critically endangered conservation status under the IUCN red list (http://www.iucnredlist.org/). Additionally, none of the 
species in this genus has been sequenced of its complete chloroplast genome. It is therefore essential to obtain the complete chloroplast genome sequence as a step towards generating genomic resources that can aid future phylogenomic studies within this family.

The chloroplast is crucial for plant cell processes such as photosynthesis [8-10] and physiological and development processes such as leaf and root development [11-13]. This organelle owns a single circular DNA, which in angiosperms is four-structured made of two duplicates of inverted repeat (IR) regions, one large single-copy (LSC) region, and one small single-copy (SSC) region [14]. Almost all chloroplast (cp) genomes studied show a relatively limited size range occurring between 120 and 160 kilobase pairs $[10,14-16]$ and comprise 110-130 genes, of which four are ribosomal RNA genes, $\sim 80$ are protein-coding, and $\sim 30$ are transfer RNAs [17].

Complete sequenced chloroplast genomes have numerically improved of late owing to the technical developments in DNA sequencing $[11,18]$ such as the Next-Generation Sequencing [19]. The availability of chloroplast genomes enables researchers to understand the evolution of the genomes, the structural organization, genes present, gene order, and the nucleotide alignment [18]. It has also made noteworthy contributions to phylogenetic studies of a significant number of plant families and determined their evolutionary links [11]. This has been made possible by the fact that chloroplast genome has a simple and stable genetic structure [20]. Over $1000 \mathrm{cp}$ genomes are already completely sequenced and explained (https://blast.ncbi.nlm.nih.gov/) since 1986 when the first chloroplast genome was sequenced in Tobacco [10]. However, since there are only three sequenced members of the family Gesneriaceae, identifying the unique features of the family's chloroplast genome can lead to deceptive estimates of species relations [21]. Thus, complete chloroplast genomes from additional members of the family are essential for comparative analysis to show structural variations among the genomes. The aims of this study were to present the first whole chloroplast genome sequence of the genus Streptocarpus and to conduct comparative analyses against close relatives.

\section{Materials and Methods}

2.1. Plant Material, DNA Extraction, Sequencing, and Assembly. Leaf samples were obtained from S. teitensis in Taita hills, Kenya, and immediately dried in silica gel [22] to preserve until DNA extraction. Voucher herbarium specimens (Voucher Number: SAJIT_006426) were deposited at the East Africa Herbarium (NMK) and Herbarium of Wuhan Botanical Garden (HIB) for future reference purposes. Total genomic DNA of one individual was extracted from $100 \mathrm{mg}$ of leaves via the MagicMag Genomic DNA Micro Kit (Sangon Biotech Co., Shanghai, China) guided by the manufacturer's instructions, after which the quality was assessed by electrophoresis on a $1 \%$ agarose gel. The complete genomic DNA was sequenced by the Illumina Hiseq 2000 Platform (Illumina, San Diego, CA), yielding 5,906,885,400 raw bases of which $5,883,619,800$ were clean bases. In addition, the raw data was recognized through BLAST (https://blast.ncbi.nlm.nih.gov/) search against Lysionotus pauciflorus (GenBank Number: KX752081) sequences since it showed the highest similarity. This produced sequence contigs which were organized and plotted against the already published plastid sequences of Lysionotus pauciflorus also via BLAST (https://blast.ncbi.nlm.nih.gov/) with default settings, in order to acquire the chloroplast genome reads. These reads were then assembled into contigs in Velvet 1.2.10 [23]. An alignment of the resulting contigs with closest chloroplast genomes of Lysionotus pauciflorus and Haberlea rhodopensis (GenBank Number: KX657870) was done via BLAST (https://blast.ncbi.nlm.nih.gov/).

2.2. Genome Annotation. The assembled cp genome was annotated using the online program Dual Organellar GenoMe Annotator (DOGMA) [17], combined with manual alterations for the doubtful start and stop codons based on comparison with homologous genes from other sequenced chloroplast genomes. The annotation of the tRNA genes was verified using tRNAscan-SE [24]. The circular cp genome map was constructed by the use of online OGDRAW program [25]. Finally, the annotated genome sequences were submitted to the NCBI GenBank under Accession Number MF596485.

2.3. Genome Comparison and Phylogenetic Analysis. The genome features such as GC contents and size of $S$. teitensis cp genome were compared with the available three chloroplast genomes in Gesneriaceae (Table 5) and 11 other species representing nine different families within the order Lamiales (Table 6), to check for similarities and variations. Furthermore, all four species of Gesneriaceae (Lysionotus pauciflorus, Haberlea rhodopensis, Dorcoceras hygrometricum [1] (previously known as Boea hygrometrica as in [26]), and Streptocarpus teitensis) were used to do a comparative study on the expansion and contraction of the IR regions. This was achieved through analysis of the four junctions, a characteristic feature of angiosperm chloroplast genomes [27], using GenBank genome files.

Chloroplast genome sequence alignment was constructed using Mauve program [28] to check the gene order and sequence similarities/variations between 15 Lamiales cp genomes as in Tables 5 and 6 . Nicotiana tabacum was added as reference genome (Figure 3) since it is considered to have the ideal angiosperm chloroplast genome structure. In order to determine the phylogenetic position of Streptocarpus teitensis, the cp genome annotation information of the species in Tables 5 and 6, together with two more Asterid species (Sinadoxa corydalifolia and Coffea arabica) included as outgroups, was obtained from the NCBI GenBank database. However, in this analysis the species Cistanche deserticola was not used since it has been recorded to have lost all the photosynthetic genes except $p s b M$. A phylogenetic tree was constructed using Maximum Likelihood (ML) analysis executed in RAxML 8.0.20 following the instructions provided in the manual [29]. A total of 67 protein-coding genes common to all the selected chloroplast genomes were used. The jModelTest 2.1.7 program [30] was used to choose the 
finest appropriate substitution models GTR + I + G (pinv $=0.47$ and gamma shape $=0.93$ ) based on the Akaike information criterion (AIC). A bootstrap study was achieved using 1000 replications.

2.4. SSR Analysis. Simple sequence repeats (SSRs) present in Streptocarpus teitensis cp genome were detected using MIcroSAtellite (MISA) identification tool (http://pgrc.ipkgatersleben.de/misa/), by setting the number of repeats to eight repeat units for mononucleotide SSRs, five repeat units for dinucleotides, and three repeat units for trinucleotides, tetranucleotides, and pentanucleotide SSRs. A comparison was then done with the other three species of Gesneriaceae (Figure 2).

\section{Results and Discussion}

3.1. Gene Content and Organization of Streptocarpus teitensis Chloroplast Genome. The whole chloroplast genome of Streptocarpus teitensis was found to be $153,207 \mathrm{bp}$ in length, encompassing a large single-copy (LSC) region of 84,103 bp, a small single-copy (SSC) region of $18,300 \mathrm{bp}$, and a pair of inverted repeats (IRA and IRB) of 25,402 bp each which separate the two regions (Figure 1). Gene annotation revealed that the cp genome of $S$. teitensis contains 116 unique genes, of which 80 are protein-coding (69\%), 32 are transfer RNAs (27.6\%), and 4 are ribosomal RNAs (3.4\%) (Table 1). This chloroplast genome maintained a constant overall GC content of $37.6 \%$ as observed previously in the family Gesneriaceae and also within the range of most completely sequenced chloroplast genomes of 30 to $40 \%$ [26]. Alike other dicot species, S. teitensis had the genes rps19 and trnH at the points of IR/LSC junctions. A total of six proteincoding genes (atpF, rpsl6, rpl2, rpoCl, ndhA, and $n d h B$ ) and six tRNA genes (trnA-UGC, trnK-UUU, trnG-GCC, trnI-GAU, trnL-UAA, and trnV-UAC) had one intron each, while $c l p P$ and $y c f 3$ had two introns each (Table 2). The LSC region housed 62 protein-coding and 23 tRNA genes while the SSC region had 11 protein-coding and one tRNA gene. Additionally, 18 genes occurred as duplicates in the IR regions of which seven are protein-coding $(r p l 2, r p l 23$, $y c f 2, n d h B, r p s 7, r p s 12$, and $y c f 1)$, seven tRNAs (trnI-CAU, trnL-CAA, trnV-GAC, trnI-GAU, trnA-UGC, trnR-ACG, and $\operatorname{trn} N-G U U)$, and the total four rRNAs. The GC content of IR regions (43.20\%) is higher than that of LSC (35.54\%) and SSC region (31.37\%) (Table 3), a phenomenon which was observed by $[31,32]$ and explained to be brought about by the increased occurrence of GC nucleotides in the four rRNA genes. The rps12 gene had the $5^{\prime}{ }_{-}$end positioned in the LSC region and the replicated $3^{\prime}{ }_{-}$end in the IR regions.

3.2. SSR Analysis. SSRs or microsatellites are tandemly repetitive DNA sequences that mostly measure $<6$ bp [33, 34], are spread all over the genome [32], and are categorized as mono-, di-, tri-, tetra-, penta-, and hexanucleotide [35]. Chloroplast SSRs (cpSSRs) are very polymorphic, reproducible, and plentiful in the genome [36] and are mainly useful in plant genetic studies [37]. A total of 196 cpSSR markers were detected in the chloroplast genome of Streptocarpus teitensis, with mononucleotides (57.1\%) being the majority, followed by the trinucleotides (33.2\%), dinucleotides and tetranucleotides (both 4.1\%), and finally the pentanucleotides (1.5\%) (Table 4). Compared to other Gesneriaceae species, Haberlea rhodopensis had the highest number of cpSSRs (214) while Dorcoceras hygrometricum had the lowest (175). In all the species, mononucleotide cpSSRs were the majority followed by the trinucleotides (Figure 2). There were no hexanucleotide repeats observed in the four studied Gesneriaceae species. Tetranucleotide repeats usually outdo the trinucleotides in number to some extent [32]. However, in this study, the trinucleotides (33.2\%) were second to mononucleotides (57.1\%). Kuang et al. [38] noted that short polyadenine (polyA or polyT) repeats are the major constituents of the simple sequence repeats occurring in the chloroplast genome, with tandem guanine $(\mathrm{G})$ or cytosine $(\mathrm{C})$ repeats being less frequent. This study had similar observations since, of the 112 mononucleotide repeats in S. teitensis, 106 were ATtype while only 6 were CG-type. The other three Gesneriaceae species exhibited this similarity since AT richness in each species was more than $50 \%$ of all the cpSSRs with Haberlea rhodopensis being the highest with $65.4 \%$.

3.3. Comparative Chloroplast Genomic Analyses. This sequence represents the fourth complete chloroplast genome to be sequenced in Gesneriaceae. When compared to the firstly sequenced representatives, Haberlea rhodopensis [8], Dorcoceras hygrometricum [26], and Lysionotus pauciflorus [39], the four chloroplast genomes are comparable in terms of their gene content, genome organization, and structure despite some slight differences such as genome size (Table 5). Lysionotus pauciflorus (153,856 bp) was found to be the most extensive, followed by Dorcoceras hygrometricum (153,493 bp) and Streptocarpus teitensis (153,207 bp), while Haberlea rhodopensis (153,099 bp) was the shortest. It was also found that Streptocarpus teitensis had the shortest IR region, a phenomenon thought to be caused by the large size of the SSC region. Furthermore, comparison to other Lamiales placed the genome size of Streptocarpus teitensis between the largest and smallest of the genomes (Table 6), which were Jasminum nudiflorum (Oleaceae) with $165,121 \mathrm{bp}$ and Cistanche deserticola (Orobanchaceae) with 102,657 bp, respectively. This inequality of the genome size can be explained, grounding the argument on the length of the LSC region as observed in earlier comparative studies on chloroplast genomes in Lamiales [32, 40].

The overall GC content between the four Gesneriaceae species was conserved (37.6-37.8\%). In the SSC region, Streptocarpus teitensis and Haberlea rhodopensis had lower GC contents (31.4 and 31.7\%, resp.). However, in the IRs the opposite was noted as Lysionotus pauciflorus and Dorcoceras hygrometricum had the lowest GC contents of 40.7\% compared to $\sim 43.3 \%$ of Streptocarpus teitensis and Haberlea rhodopensis. Among Lamiales (Table 6), Cistanche deserticola had the lowest GC content (36.8\%) while Andrographis paniculata and Tanaecium tetragonolobum had the highest (38.3\%).

The complete genome alignment using Mauve software was done between 15 species of Lamiales (Tables 5 and 6) and Nicotiana tabacum (Figure 3) which was added as 
TABLE 1: The functional classification of genes found in Streptocarpus teitensis chloroplast genome.

\begin{tabular}{|c|c|c|}
\hline Function & Group of genes & Gene names \\
\hline \multirow{6}{*}{ Photosynthesis } & Photosystem 1 & $p s a A, p s a B, p s a C, p s a I, p s a J$ \\
\hline & Photosystem 11 & $\begin{array}{l}p s b A, p s b B, p s b C, p s b D, p s b E, p s b F, p s b H, p s b I, p s b J, p s b K, p s b L \\
p s b M, p s b N, p s b T, p s b Z\end{array}$ \\
\hline & NADH dehydrogenase & $\begin{array}{l}n d h A^{*}, n d h B^{*}(x 2), n d h C, n d h D, n d h E, n d h F, n d h G, n d h H, n d h I, \\
n d h J, n d h K\end{array}$ \\
\hline & ATP synthase & atpA, atpB, atpE, atpF $F^{*}$, atpH, atpI \\
\hline & Cytochrome b/f complex & pet $A$, pet $B$, petD, petG, pet $L$, pet $N$ \\
\hline & RubisCO large subunit & $r b c L$ \\
\hline \multirow{8}{*}{ Self-replication } & RNA polymerase & rpoA, rроB, rpoC1* ${ }^{*}$, rpoC2 \\
\hline & $\begin{array}{l}\text { Ribosomal proteins (Large } \\
\text { Sub-unit) }\end{array}$ & rpl2*, rpl14, rpl16, rpl20, rpl22, rpl23(x2), rpl32, rpl33, rpl36 \\
\hline & Ribosomal proteins (small subunit) & $\begin{array}{l}\text { rps2, rps3, rps4, rps7(x2), rps8, rps11, rps12(x2), rps14, rps15, rps16 } \\
\text { rps18, rps19 }\end{array}$ \\
\hline & Ribosomal RNAs & $r r n 4.5(x 2), r r n 5(x 2), r r n 16(x 2), r r n 23(x 2)$ \\
\hline & \multirow{4}{*}{ Transfer RNAs } & $\begin{array}{l}\operatorname{trn} A-U G C^{*}(x 2), \operatorname{trn} C-G C A, \operatorname{trn} D-G U C, \operatorname{trn} E-U U C, \operatorname{trn} F-G A A, \\
\operatorname{trn} G-G C C^{*}, \operatorname{trn} G-U C C, \operatorname{trn} H-G U G\end{array}$ \\
\hline & & $\begin{array}{l}\operatorname{trnI}-C A U(x 2), \operatorname{trn} I-G A U^{*}(x 2), \operatorname{trnK}-U U U^{*}, \operatorname{trn} L-C A A(x 2) \\
\operatorname{trnL}-U A A^{*}, \operatorname{trnL}-U A G, \operatorname{trnfM-CAU}\end{array}$ \\
\hline & & $\begin{array}{l}\operatorname{trnN}-G U U(x 2), \operatorname{trn} P-U G G, \operatorname{trn} Q-U U G, \operatorname{trn} R-A C G(x 2), \operatorname{trn} R-U C U, \\
\operatorname{trn} S-G C U, \operatorname{trn} S-G G A, \operatorname{trn} S-U G A\end{array}$ \\
\hline & & $\begin{array}{l}\operatorname{trn} T-G G U, \operatorname{trn} T-U G U, \operatorname{trn} V-G A C(x 2), \operatorname{trn} V-U A C^{*}, \operatorname{trnW}-C C A, \\
\operatorname{trn} Y-G U A, \operatorname{trn} P-G G G, \operatorname{trn} M-C A U\end{array}$ \\
\hline Proteins of unknown function & & $y c f 1(x 2), y c f 2(x 2), y c f 3^{* *}, y c f 4$ \\
\hline \multirow{6}{*}{ Other genes } & Protease & $c l p P^{* *}$ \\
\hline & Maturase & matK \\
\hline & Translational initiation factor & $\operatorname{infA}$ \\
\hline & Envelope membrane protein & $\operatorname{cemA}$ \\
\hline & Subunit of acetyl-CoA-carboxylase & $a c c D$ \\
\hline & c-type cytochrome synthesis & $\operatorname{ccs} A$ \\
\hline
\end{tabular}

* marks genes with one intron; ** marks genes with two introns; $(x 2)$ shows genes with duplicates.

TABLE 2: The genes with introns in the Streptocarpus teitensis chloroplast genome and the length of the exons and introns.

\begin{tabular}{|c|c|c|c|c|c|c|}
\hline Gene & Region & Exon $1(\mathrm{bp})$ & Intron 1 (bp) & Exon 2 (bp) & Intron 2 (bp) & Exon 3 (bp) \\
\hline atpF & LSC & 472 & 633 & 144 & & \\
\hline rps16 & LSC & 207 & 1636 & 48 & & \\
\hline rpl2 & IR & 435 & 670 & 393 & & \\
\hline rpoC1 & LSC & 1620 & 784 & 456 & & \\
\hline$n d h A$ & SSC & 540 & 1069 & 552 & & \\
\hline$n d h B$ & IR & 756 & 679 & 777 & & \\
\hline $\operatorname{trn} A-U G C$ & IR & 38 & 834 & 35 & & \\
\hline $\operatorname{trnI}-G A U$ & IR & 35 & 935 & 42 & & \\
\hline $\operatorname{trnK-UUU}$ & LSC & 35 & 2493 & 37 & & \\
\hline $\operatorname{trn} G-G C C$ & LSC & 23 & 704 & 37 & & \\
\hline $\operatorname{trnL} L-U A A$ & LSC & 37 & 469 & 50 & & \\
\hline $\operatorname{trnV} V-U A C$ & LSC & 37 & 575 & 38 & & \\
\hline$y c f 3$ & LSC & 150 & 712 & 228 & 688 & 129 \\
\hline$C l p P$ & LSC & 234 & 631 & 297 & 819 & 69 \\
\hline
\end{tabular}




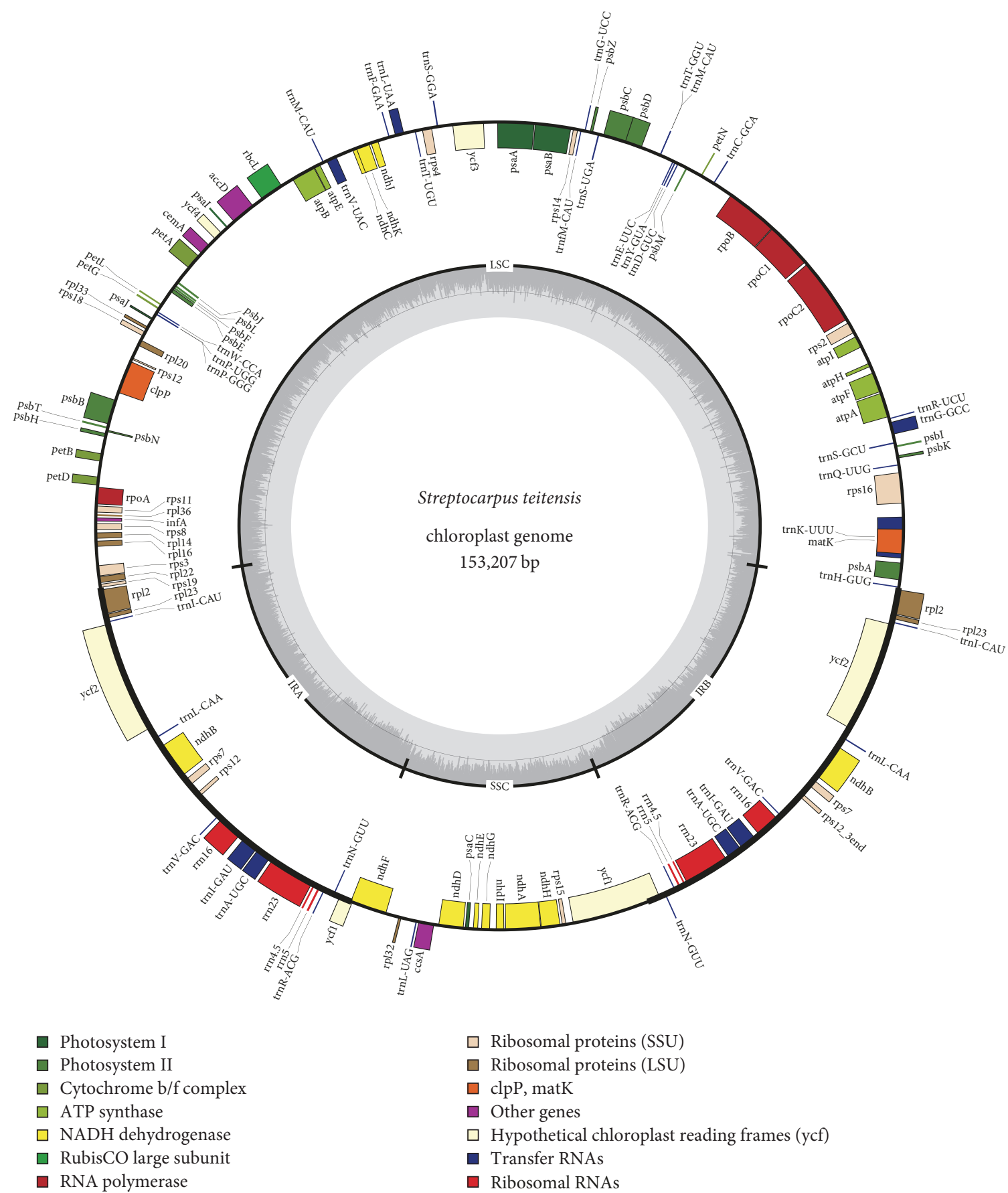

FIGURE 1: The gene map of the chloroplast genome of Streptocarpus teitensis. Genes drawn inside the map are transcribed clockwise, while genes drawn outside are transcribed counterclockwise. Different colors represent genes of different functional groups. Inverted repeats (IRA and IRB) are marked by the dark bold lines; GC and AT contents are, respectively, represented by the dark and light grey colors inside the map.

TABLE 3: The AT and GC\% in different regions of Streptocarpus teitensis cp genome.

\begin{tabular}{lcccccc}
\hline Region & Length (bp) & A (\%) & T (\%) & G (\%) & C (\%) & GC (\%) \\
\hline LSC & 84,103 & 31.57 & 32.88 & 17.36 & 18.18 & 35.54 \\
SSC & 18,300 & 34.13 & 34.5 & 15.15 & 31.37 \\
IRA & 25,402 & 28.46 & 28.34 & 22.43 & 20.77 & 43.2 \\
IRB & 25,402 & 28.34 & 28.45 & 20.77 & 22.43 & 43.2 \\
Total genome & 153,207 & 30.82 & 31.59 & 18.5 & 19.08 \\
\hline
\end{tabular}




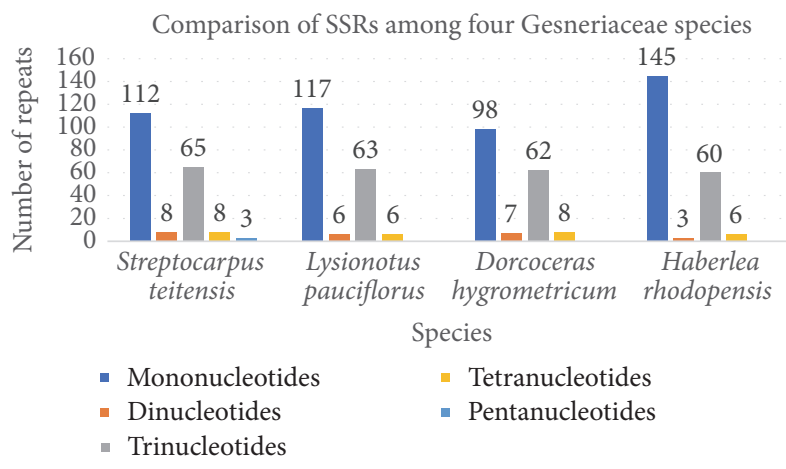

(a)

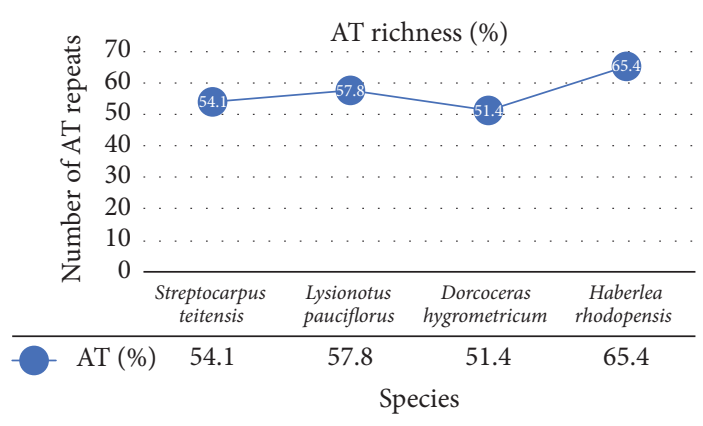

(b)

FIGURE 2: Comparison of SSR repeats (a) and AT repeats richness (b) among four Gesneriaceae species.

TABLE 4: Number of SSR repeats in Streptocarpus teitensis chloroplast genome.

\begin{tabular}{|c|c|c|c|c|c|c|c|c|c|c|c|c|}
\hline Repeat sequences & Number of repeats & & & & & & & & & & & Total \\
\hline & 3 & 4 & 5 & 6 & 7 & 8 & 9 & 10 & 11 & 12 & 13 & \\
\hline $\mathrm{A} / \mathrm{T}$ & & & & & & 55 & 33 & 8 & 5 & 4 & 1 & 106 \\
\hline $\mathrm{C} / \mathrm{G}$ & & & & & & 4 & 2 & & & & & 6 \\
\hline $\mathrm{AG} / \mathrm{CT}$ & & & 2 & & & & & & & & & 2 \\
\hline $\mathrm{AT} / \mathrm{AT}$ & & & 4 & 1 & & 1 & & & & & & 6 \\
\hline AAC/GTT & 10 & & & & & & & & & & & 10 \\
\hline AAG/CTT & 20 & 1 & & & & & & & & & & 21 \\
\hline AAT/ATT & 18 & 1 & & & & & & & & & & 19 \\
\hline AGC/GCT & 6 & & & & & & & & & & & 6 \\
\hline AGG/CCT & 4 & & & & & & & & & & & 4 \\
\hline ATC/GAT & 5 & & & & & & & & & & & 5 \\
\hline AAAT/ATTT & 3 & & & & & & & & & & & 3 \\
\hline AATC/GATT & 2 & & & & & & & & & & & 2 \\
\hline AATT/AATT & 1 & & & & & & & & & & & 1 \\
\hline AGAT/ATCT & 2 & & & & & & & & & & & 2 \\
\hline AAAAG/CTTTT & 2 & & & & & & & & & & & 2 \\
\hline AATTC/GAATT & 1 & & & & & & & & & & & 1 \\
\hline Total & & & & & & & & & & & & 196 \\
\hline
\end{tabular}

reference genome since it is considered to have an ancestral arrangement of genes in angiosperms [41]. It was observed that many genome regions were conserved, with few variations in gene order, gene loss/gain events, and the direction in which the genes were transcribed. The inverted repeat regions ( 90,000-110,000; 130,000-160,000) showed the highest variations among the aligned chloroplast genomes, while the LSC (1- 80,000) and SSC ( 110,000-130,000) were the most comparable. In addition, two species (Cistanche deserticola and Jasminum nudiflorum) had inversion events. Cistanche deserticola was the most notably different from other Lamiales, with all genes inversed and a reduction in genome size. The reduction in genome size was observed in a previous study and attributed to the loss of photosynthetic genes apart from psbM [42]. The inversion in Jasminum nudiflorum occurred on two genes psaI and $y c f 4$. The four Gesneriaceae species in this study had a conserved gene arrangement and order and similar to other Lamiales as signified by the color blocks, with few variations in the sizes of the gene classes, drawing a conclusion of a close and conservative evolution of Gesneriaceae in the order Lamiales.

3.4. IR Expansion and Contraction. The IR regions have been observed to be potential distinguishing features among most angiosperms, as their expansions or contractions in or out of the single-copy regions are attributed to the different chloroplast genome sizes $[43,44]$. The order in which genes are arranged in the junctions between the genome regions has also differentiated between species [45]. A comparable observation was made in photosynthetic orchids, whereby the chloroplast genomes exposed comparable structures but the IR and single-copy regions intersections together with the $n d h$ genes displayed some variations [46]. Palmer and Thompson [47] discovered that chloroplast genomes which have lost the IR regions tend to be rearranged, findings which were duplicated by Strauss et al. [48], signifying that these regions function to uphold the structure of the genome. However, from both studies, it was not clear whether IR loss 
TABLE 5: Comparison of the features of four Gesneriaceae chloroplast genomes.

\begin{tabular}{lcccc}
\hline Feature & Streptocarpus teitensis & Dorcoceras hygrometricum & Lysionotus pauciflorus & Haberlea rhodopensis \\
\hline Genome size (bp) & 153,207 & 153,493 & 153,856 & 153,099 \\
Large single copy (bp) & 84,103 & 84,692 & 85,087 & 84,443 \\
Small single copy (bp) & 18,300 & 17,901 & 17,839 & 17,826 \\
Inverted repeats (bp) & 25,402 & 25,450 & 25,465 & 25,415 \\
GC content in LSC (\%) & 35.5 & 35.6 & 35.4 & 35.7 \\
GC content in SSC (\%) & 31.4 & 36.4 & 36.6 & 31.7 \\
GC content in IR (\%) & 43.2 & 40.7 & 40.6 & 43.3 \\
Overall AT content (\%) & 62.4 & 62.4 & 62.5 & 62.2 \\
Overall GC content (\%) & 37.6 & 37.6 & 37.5 & 37.8 \\
\hline
\end{tabular}

TABLE 6: Comparison of the features of Streptocarpus teitensis with other 11 Lamiales chloroplast genomes.

\begin{tabular}{|c|c|c|c|c|c|c|c|}
\hline Species & Family & LSC (bp) & SSC (bp) & IR (bp) & Total (bp) & CG content $\%$ & Accession number \\
\hline Streptocarpus teitensis & Gesneriaceae & 84,103 & 18,300 & 25,402 & 153,207 & 37.58 & MF596485 \\
\hline Cistanche deserticola & Orobanchaceae & 49,130 & 8,819 & 22,354 & 102,657 & 36.8 & KC128846 \\
\hline Sesamum indicum & Pedaliaceae & 85,170 & 17,872 & 25,141 & 153,324 & 38 & JN637766 \\
\hline Premna microphylla & Lamiaceae & 86,078 & 17,689 & 25,763 & 155,293 & 37.9 & KM981744 \\
\hline Salvia miltiorrhiza & Lamiaceae & 82,695 & 17,555 & 25,539 & 151,328 & 38 & JX312195 \\
\hline Origanum vulgare subsp. vulgare & Lamiaceae & 83,136 & 17,745 & 25,527 & 151,935 & 38 & JX880022 \\
\hline Utricularia foliosa & Lentibulariaceae & 82,720 & 17,481 & 25,325 & 150,851 & 37.32 & KY025562 \\
\hline Andrographis paniculata & Acanthaceae & 82,459 & 17,190 & 25,300 & 150,249 & 38.3 & NC022451 \\
\hline Tanaecium tetragonolobum & Bignoniaceae & 84,612 & 17,586 & 25,789 & 153,776 & 38.3 & KR534325 \\
\hline Paulownia coreana & Paulowniaceae & 85,241 & 17,736 & 25,784 & 154,545 & 38 & KP718622 \\
\hline Scrophularia dentata & Scrophulariaceae & 84,058 & 17,449 & 25,523 & 152,553 & 38 & KT428154 \\
\hline Jasminum nudiflorum & Oleaceae & 92,877 & 13,272 & 29,486 & 165,121 & 38 & NC_008407 \\
\hline
\end{tabular}

induced genome rearrangements and the latter study concluded that IR loss might reduce the resistance of the genome to rearrangements. A later study by Chumley et al. [49] found Pelargonium $x$ hortorum genome to be reorganized despite containing the IR regions, further suggesting that IR loss does not induce genome rearrangements.

Comparing the LSC-IR-SSC junctions and their adjacent genes between Streptocarpus teitensis, Lysionotus pauciflorus, Dorcoceras hygrometricum, and Haberlea rhodopensis revealed some notable variations (Figure 4). The LSC-IRA junction had expanded into the gene rps19 in two species (Lysionotus pauciflorus, $36 \mathrm{bp}$, and Dorcoceras hygrometricum, $37 \mathrm{bp}$ ) while in Streptocarpus teitensis and Haberlea rhodopensis the gene was $3 \mathrm{bp}$ and $108 \mathrm{bp}$ away from the junction, respectively. This resulted in the two species (Lysionotus pauciflorus and Dorcoceras hygrometricum) having a pseudogene of the rps19 of 36 and 37 base pairs, respectively, at the IRB-LSC junction. A pseudogenized $y c f 1$ occurred at the IRASSC junctions in all species as a result of the extension of SSCIRB junction into the $y c f 1$ gene, with variable extensions of the gene into the SSC region observed in the four species. An overlap of $\Psi y c f 1$ and $n d h F$ genes was observed in Dorcoceras hygrometricum (121 bp), Lysionotus pauciflorus (137 bp), and Haberlea rhodopensis (11 bp) while in Streptocarpus teitensis the two genes joined each other. There was similarity in the SSC-IRB junction, in which $y c f 1$ gene occurred in all species, although the sizes varied with Streptocarpus teitensis having the largest $(5,489 \mathrm{bp})$ and Haberlea rhodopensis the smallest (5,430 bp). A previous study on Rosaceae [27] observed the IRB-LSC junction to be characterized by the genes rpl2 (IRB) and trnH-GUG (LSC). Similar observations were made in two Gesneriaceae species under the present study, while the species Lysionotus pauciflorus and Dorcoceras hygrometricum had a pseudogene of rps19. Additionally, the IRB-LSC junction occurred away from the gene trnH-GUG at variable lengths (3-44 base pairs) in the four species.

3.5. Phylogenetic Analysis. The accessibility of numerous complete genomes sequenced has paved way for phylogenomics, a new method which has been observed to significantly offer solution to evolutionary issues by use of many characters $[50,51]$ contrary to the original phylogenetics. Previously, complete chloroplast genomes have helped resolve identity issues in the basal families of angiosperms [52]. In the Maximum Likelihood tree, 11 of the total 14 nodes had observed bootstrap values of $\geq 95 \%$, with 10 of these having bootstrap values of $100 \%$, while only three had low bootstrap values. The outcome displayed that $S$. teitensis clustered more closely with Lysionotus pauciflorus and Dorcoceras hygrometricum than with Haberlea rhodopensis (Figure 5). Additionally, at the order level, the family Gesneriaceae closely allied to family Scrophulariaceae. Generally, all the 14 species formed a lineage (Lamiales) noticeably distinct from the two out-group species. 


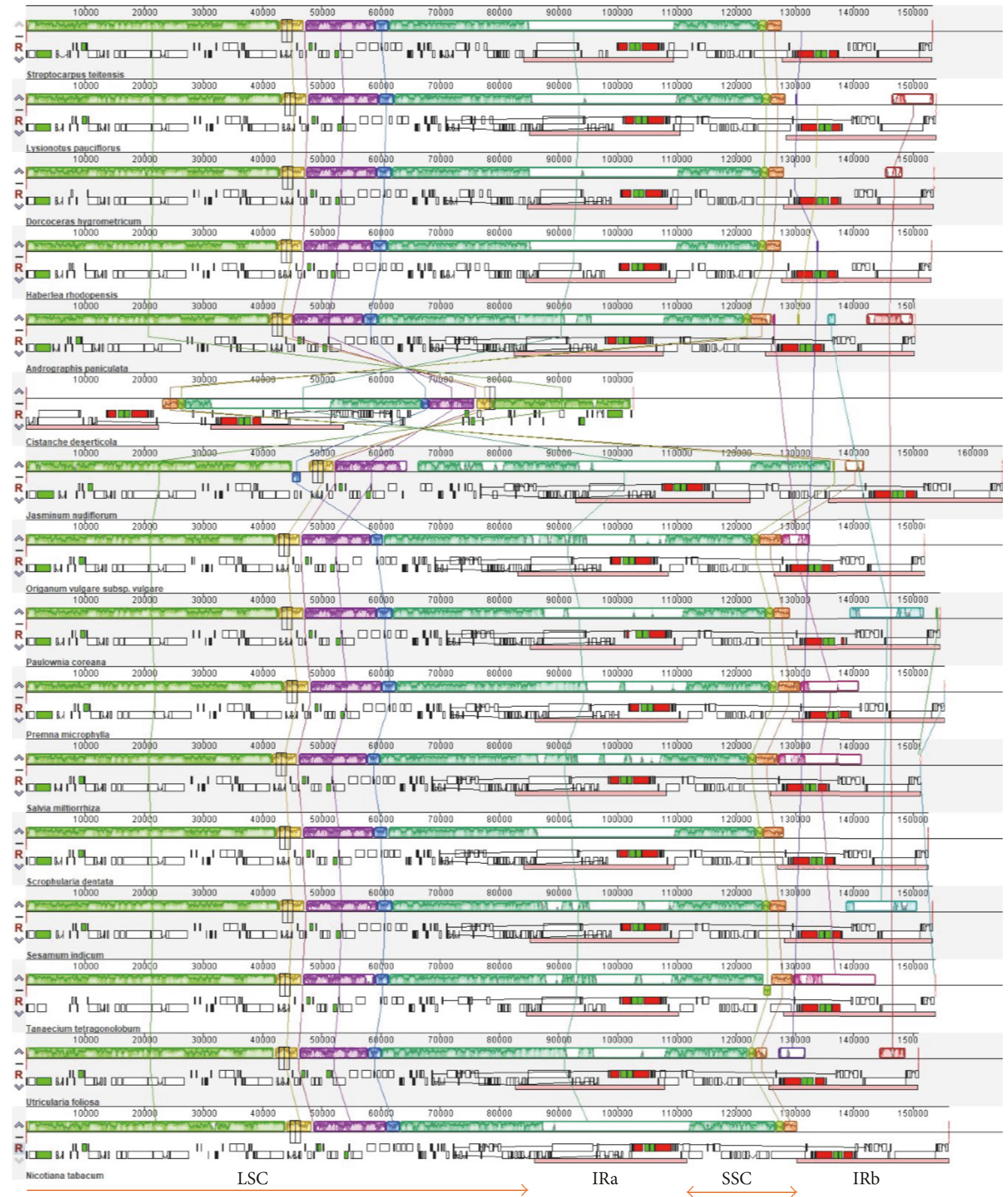

Figure 3: Mauve multiple alignment of 15 Lamiales, with Nicotiana tabacum set as the reference genome. Color-coded segments indicate regions that shared same genes across different species' genomes. The extent of sequence similarities is indicated by the colored parts inside each region. Lines connect regions with homologous sequences among two genomes.

\section{Conclusion}

Gesneriaceae is one of the important families in the order Lamiales as it is traded for its attractive flowers but their chloroplast genomes are understudied. Our research described the complete cp genome of Streptocarpus teitensis, a critically endangered species with one surviving population. This was the first whole cp genome to be reported in the genus
Streptocarpus and the fourth in the family Gesneriaceae. The $\mathrm{cp}$ genome revealed genetic features and arrangement typical of the angiosperm cp genome. It also comprised 116 unique genes of which 80 are protein-coding, 32 tRNAs, and 4 rRNAs. Comparisons of the LSC/IR/SSC junctions in Gesneriaceae exposed some outstanding differences in the gene arrangements. Study of the phylogenetic tree revealed that Lysionotus pauciflorus and Dorcoceras hygrometricum were 


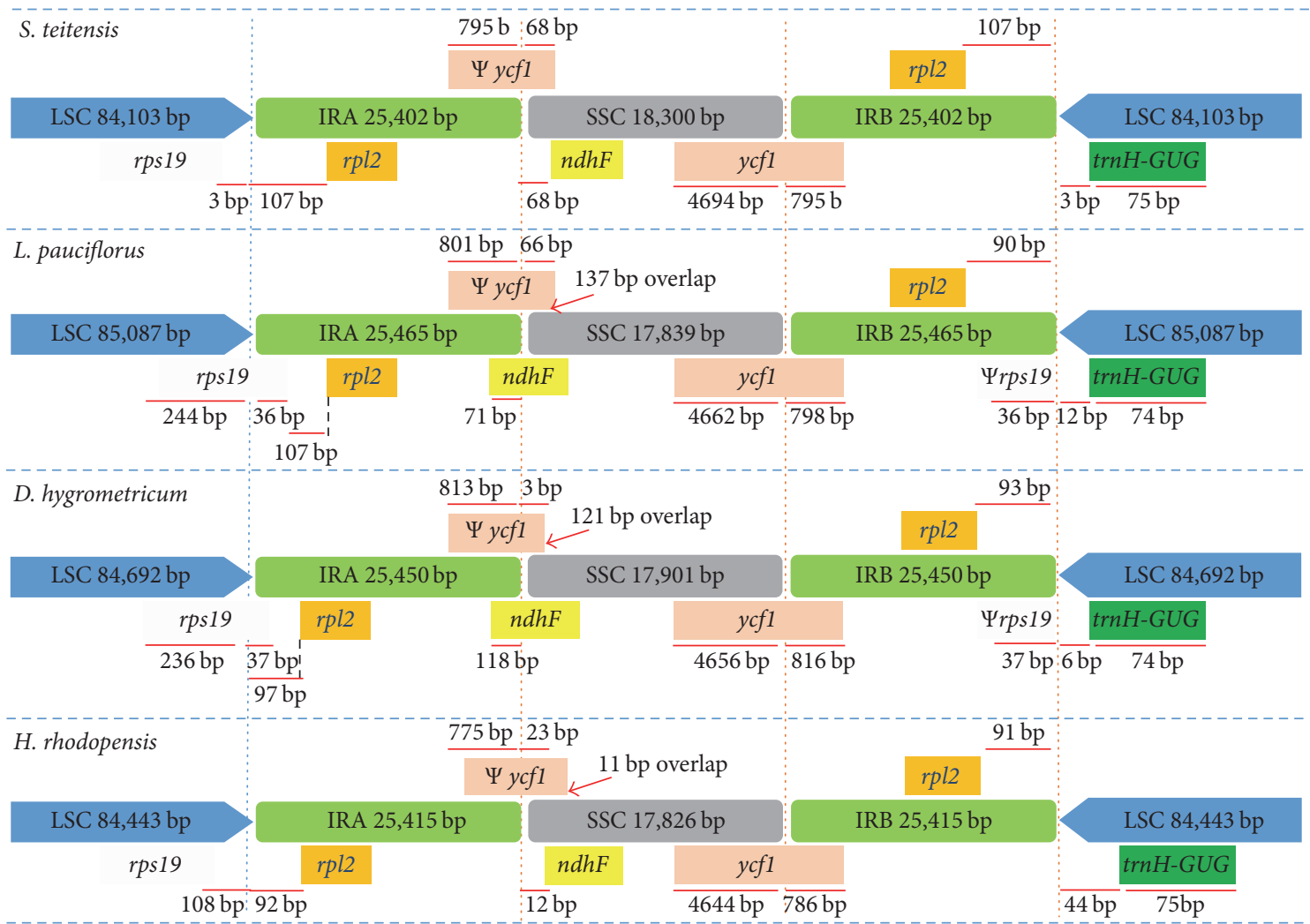

FIGURE 4: Comparison of LSC, SSC, and IR junctions among Gesneriaceae species. $\Psi$ indicates a pseudogene. Genes above the regions are direct while genes below are reverse.

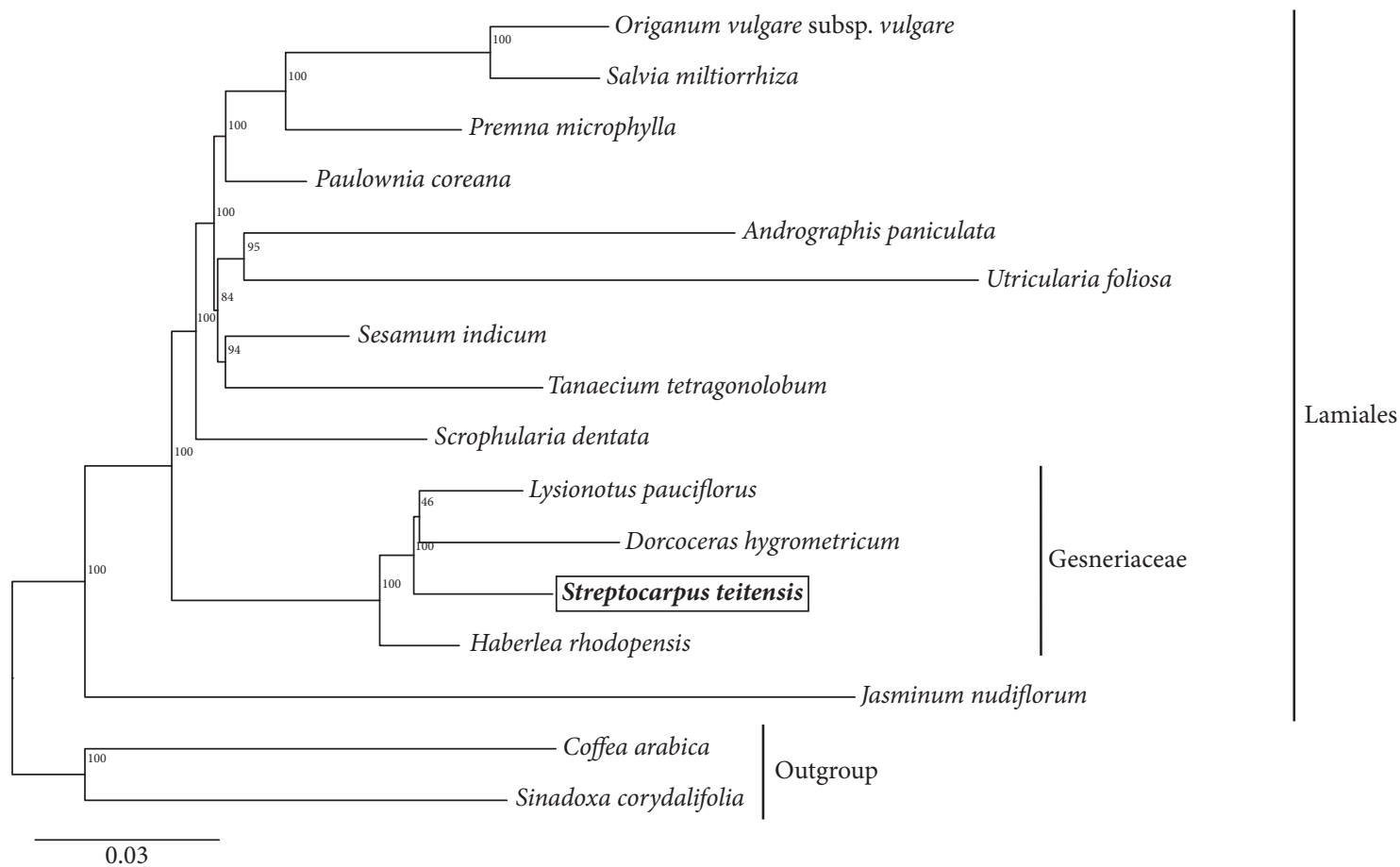

FIGURE 5: Maximum Likelihood phylogenetic tree of 14 species of Lamiales, and two other Asterid species as outgroups based on 67 chloroplast genome protein-coding genes' sequences. 
closer to Streptocarpus teitensis than Haberlea rhodopensis, while the family Scrophulariaceae was close to Gesneriaceae. This research informs the genetic structure of this endangered species and compares it to other members of the family and the order Lamiales.

\section{Disclosure}

The funders played no role in the study design, data collection and analysis, decision to publish, or preparation of the manuscript.

\section{Conflicts of Interest}

The authors affirm no conflicts of interest.

\section{Authors' Contributions}

Guang-Wan $\mathrm{Hu}$, Itambo Malombe, and Qing-Feng Wang conceived and designed the experiments. Cornelius M. Kyalo and Andrew W. Gichira performed the experiments, analyzed the data, wrote the paper, and prepared the figures and tables. Zhi-Zhong Li and Josphat K. Saina analyzed the data and reviewed the draft of the paper. Qing-Feng Wang conceived the experiment, contributed reagents/ materials/analysis tools, and reviewed the drafts of the paper. Cornelius M. Kyalo wrote the paper.

\section{Acknowledgments}

The authors express their gratitude to David Melly, Solomon Kipkoech, and Benjamin Muema for providing fieldwork assistance and Justus Mulinge for proofreading and editing. They also acknowledge Chen Jin-Ming for useful comments on this manuscript. This work was supported by the University of Chinese Academy of Sciences (UCAS), Wuhan Botanical Garden (WBG), and Sino-Africa Joint Research Center (SAJOREC) under the Identification Numbers Y323771W07 and SAJC201322.

\section{References}

[1] C. Puglisi, T. L. Yao, R. Milne, M. Möller, and D. J. Middleton, "Generic recircumscription in the Loxocarpinae (Gesneriaceae), as inferred by phylogenetic and morphological data," TAXON, vol. 65, no. 2, pp. 277-292, 2016.

[2] M. J. de Villiers, M. D. Pirie, M. Hughes, M. Möller, T. J. Edwards, and D. U. Bellstedt, "An approach to identify putative hybrids in the 'coalescent stochasticity zone', as exemplified in the African plant genus Streptocarpus (Gesneriaceae)," New Phytologist, vol. 198, no. 1, pp. 284-300, 2013.

[3] M. Hughes, M. Möller, D. U. Bellstedt, T. J. Edwards, and M. Woodhead, "EST and random genomic nuclear microsatellite markers for Streptocarpus," Molecular Ecology Resources (Formerly known as Molecular Ecology Notes), vol. 4, no. 1, pp. 36-38, 2004.

[4] M. Möller and Q. C. B. Cronk, "Phylogenetic studies in Streptocarpus (Gesneriaceae): Reconstruction of biogeographic history and distribution patterns," Systematics and Geography of Plants, vol. 71, no. 2, pp. 545-555, 2001.
[5] M. Moller and Q. C. B. Cronk, "Phylogeny and disjunct distribution: Evolution of Saintpaulia (Gesneriaceae)," Proceedings of the Royal Society B Biological Science, vol. 264, no. 1389, pp. 1827-1836, 1997.

[6] K. Nishii, M. Hughes, M. Briggs et al., "Streptocarpus redefined to include all afro-malagasy gesneriaceae: Molecular phylogenies prove congruent with geographical distribution and basic chromosome numbers and uncover remarkable morphological homoplasies," TAXON, vol. 64, no. 6, pp. 1243-1274, 2015.

[7] A. Eastwood, B. Bytebier, H. Tye, A. Tye, A. Robertson, and M. Maunder, "The conservation status of Saintpaulia," Curtis\&apos;s Botanical Magazine, vol. 15, no. 1, pp. 49-62, 1998.

[8] Z. Ivanova, G. Sablok, E. Daskalova et al., "Chloroplast genome analysis of resurrection tertiary relict Haberlea rhodopensis highlights genes important for desiccation stress response," Frontiers in Plant Science, vol. 8, article no. 204, 2017.

[9] G. Raman and S. Park, "Analysis of the complete chloroplast genome of a medicinal plant, Dianthus superbus var. longicalyncinus, from a comparative genomics perspective," PLoS ONE, vol. 10, no. 10, article e0141329, 2015.

[10] K. Shinozaki, M. Ohme, M. Tanaka et al., "The complete nucleotide sequence of the tobacco chloroplast genome," Plant Molecular Biology Reporter, vol. 4, no. 3, pp. 111-148, 1986.

[11] D. Henry, L. Choun-Sea, Y. Ming, and C. Wan-Jung, "Chloroplast genomes: diversity, evolution, and applications in genetic engineering," Genome Biology, vol. 17, article 134, 2016.

[12] G. Horiguchi, A. Mollá-Morales, J. M. Pérez-Pérez et al., "Differential contributions of ribosomal protein genes to arabidopsis thaliana leaf development," The Plant Journal, vol. 65, no. 5, pp. 724-736, 2011.

[13] T. Inaba and Y. Ito-Inaba, "Versatile roles of plastids in plant growth and development," Plant \& Cell Physiology (PCP), vol. 51, no. 11, pp. 1847-1853, 2010.

[14] J. D. Palmer, "Comparative organization of chloroplast genomes," Annual Review of Genetics, vol. 19, no. 1, pp. 325-354, 1985.

[15] S. Wicke, G. M. Schneeweiss, C. W. dePamphilis, K. F. Müller, and D. Quandt, "The evolution of the plastid chromosome in land plants: Gene content, gene order, gene function," Plant Molecular Biology, vol. 76, no. 3-5, pp. 273-297, 2011.

[16] C.-F. Jheng, T.-C. Chen, J.-Y. Lin, T.-C. Chen, W.-L. Wu, and C.-C. Chang, "The comparative chloroplast genomic analysis of photosynthetic orchids and developing DNA markers to distinguish Phalaenopsis orchids," Journal of Plant Sciences, vol. 190, pp. 62-73, 2012.

[17] S. K. Wyman, R. K. Jansen, and J. L. Boore, "Automatic annotation of organellar genomes with DOGMA," Bioinformatics, vol. 20, no. 17, pp. 3252-3255, 2004.

[18] L. Gao, X. Yi, Y.-X. Yang, Y.-J. Su, and T. Wang, "Complete chloroplast genome sequence of a tree fern Alsophila spinulosa: insights into evolutionary changes in fern chloroplast genomes," BMC Evolutionary Biology, vol. 9, no. 1, article 130, 2009.

[19] E. R. Mardis, "Next-generation sequencing platforms," Annual Review of Analytical Chemistry, vol. 6, pp. 287-303, 2013.

[20] W. Dong, J. Liu, J. Yu, L. Wang, S. Zhou, and A. Moustafa, "Highly Variable Chloroplast Markers for Evaluating Plant Phylogeny at Low Taxonomic Levels and for DNA Barcoding," PLoS ONE, vol. 7, no. 4, p. e35071, 2012.

[21] J. Leebens-Mack, L. A. Raubeson, L. Cui et al., "Identifying the basal angiosperm node in chloroplast genome phylogenies: Sampling one's way out of the Felsenstein zone," Molecular Biology and Evolution, vol. 22, no. 10, pp. 1948-1963, 2005. 
[22] M. W. Chase and H. H. Hills, "Silica Gel: An Ideal Material for Field Preservation of Leaf Samples for DNA Studies," TAXON, vol. 40, no. 2, p. $215,1991$.

[23] D. R. Zerbino and E. Birney, "Velvet: algorithms for de novo short read assembly using de Bruijn graphs," Genome Research, vol. 18, no. 5, pp. 821-829, 2008.

[24] P. Schattner, A. N. Brooks, and T. M. Lowe, "The tRNAscanSE, snoscan and snoGPS web servers for the detection of tRNAs and snoRNAs," Nucleic Acids Research, vol. 33, no. 2, pp. W686W689, 2005.

[25] M. Lohse, O. Drechsel, S. Kahlau, and R. Bock, "OrganellarGenomeDRAW - a suite of tools for generating physical maps of plastid and mitochondrial genomes and visualizing expression data sets," Nucleic Acids Research, vol. 41, pp. W575W581, 2013.

[26] T. Zhang, Y. Fang, X. Wang et al., "The complete chloroplast and mitochondrial genome sequences of boea hygrometrica: insights into the evolution of plant organellar genomes," PLoS ONE, vol. 7, no. 1, Article ID e30531, 2012.

[27] A. W. Gichira, Z. Li, J. K. Saina et al., "The complete chloroplast genome sequence of an endemic monotypic genus Hagenia (Rosaceae): Structural comparative analysis, gene content and microsatellite detection," PeerJ, vol. 2017, no. 1, Article ID e2846, 2017.

[28] A. E. Darling, B. Mau, and N. T. Perna, "Progressivemauve: multiple genome alignment with gene gain, loss and rearrangement," PLoS ONE, vol. 5, no. 6, Article ID el1147, 2010.

[29] A. Stamatakis, "RAxML version 8: a tool for phylogenetic analysis and post-analysis of large phylogenies," Bioinformatics, vol. 30, no. 9, pp. 1312-1313, 2014.

[30] D. Darriba, G. L. Taboada, R. Doallo, and D. Posada, "JModelTest 2: more models, new heuristics and parallel computing," Nature Methods, vol. 9, no. 8, p. 772, 2012.

[31] Z. Wu, C. Gu, L. R. Tembrock, D. Zhang, and S. Ge, "Characterization of the whole chloroplast genome of Chikusichloa mutica and its comparison with other rice tribe (Oryzeae) species," PLoS ONE, vol. 12, no. 5, Article ID e0177553, 2017.

[32] J. Qian, J. Song, H. Gao et al., "The Complete Chloroplast Genome Sequence of the Medicinal Plant Salvia miltiorrhiza," PLoS ONE, vol. 8, no. 2, Article ID e57607, 2013.

[33] M.-F. Deguilloux, M.-H. Pemonge, and R. J. Petit, "Use of chloroplast microsatellites to differentiate oak populations," Annals of Forest Science, vol. 61, no. 8, pp. 825-830, 2004.

[34] J. Kang, J. Lu, S. Qiu, Z. Chen, J. Liu, and H. Wang, "Dendrobium SSR markers play a good role in genetic diversity and phylogenetic analysis of Orchidaceae species," Scientia Horticulturae, vol. 183, pp. 160-166, 2015.

[35] M. Wang, A. Barkley Noelle, and J. T. M, "Microsatellite markers in plants and Insects," Applications of Biotechnology, Part 1, no. Special Issue 1, pp. 54-67, 2009.

[36] T. Kaila, P. K. Chaduvla, H. C. Rawal et al., "Chloroplast genome sequence of clusterbean (Cyamopsis tetragonoloba L.): Genome structure and comparative analysis," Gene, vol. 8, no. 9, article no. 212, 2017.

[37] B. Zhang, H.-W. Wang, Y.-Q. Cheng, Y.-Z. Ye, and Z.-S. Wang, "Microsatellite markers for Dayaoshania cotinifolia (Gesneriaceae), a critically endangered perennial herb," American Journal of Botany, vol. 98, no. 9, pp. e256-e258, 2011.

[38] D.-Y. Kuang, H. Wu, Y.-L. Wang, L.-M. Gao, S.-Z. Zhang, and L. Lu, "Complete chloroplast genome sequence of Magnolia kwangsiensis (Magnoliaceae): Implication for DNA barcoding and population genetics," Genome, vol. 54, no. 8, pp. 663-673, 2011.

[39] T. Ren, W. Zheng, K. Han, S. Zeng, J. Zhao, and Z.-L. Liu, "Characterization of the complete chloroplast genome sequence of Lysionotus pauciflorus (Gesneriaceae)," Conservation Genetics Resources, vol. 9, no. 2, pp. 185-187, 2017.

[40] L. Ni, Z. Zhao, G. D. Dorje, and M. Ma, "The complete chloroplast genome of Ye-Xing-Ba (Scrophularia dentata; Scrophulariaceae), an alpine tibetan herb," PLoS ONE, vol. 11, no. 7, Article ID e0158488, 2016.

[41] L. Yan, X. Lai, X. Li, C. Wei, X. Tan, and Y. Zhang, "Analyses of the complete genome and gene expression of chloroplast of sweet potato [Ipomoea batata]," PLoS ONE, vol. 10, no. 4, Article ID e012083, 2015.

[42] X. Li, T.-C. Zhang, Q. Qiao et al., "Complete Chloroplast Genome Sequence of Holoparasite Cistanche deserticola (Orobanchaceae) Reveals Gene Loss and Horizontal Gene Transfer from Its Host Haloxylon ammodendron (Chenopodiaceae)," PLoS ONE, vol. 8, no. 3, Article ID e58747, 2013.

[43] G. M. Plunkett and S. R. Downie, "Expansion and contraction of the chloroplast inverted repeat in Apiaceae subfamily Apioideae," Systematic Botany, vol. 25, no. 4, pp. 648-667, 2000.

[44] H. Zhang, N. Hall, J. S. McElroy, E. K. Lowe, and L. R. Goertzen, "Complete plastid genome sequence of goosegrass (Eleusine indica) and comparison with other Poaceae," Gene, vol. 600, pp. 36-43, 2017.

[45] J.-B. Yang, M. Tang, H.-T. Li, Z.-R. Zhang, and D.-Z. Li, "Complete chloroplast genome of the genus Cymbidium: Lights into the species identification, phylogenetic implications and population genetic analyses," BMC Evolutionary Biology, vol. 13, no. 1, article no. 84, 2013.

[46] J. Luo, B.-W. Hou, Z.-T. Niu, W. Liu, Q.-Y. Xue, and X.-Y. Ding, "Comparative chloroplast genomes of photosynthetic orchids: Insights into evolution of the Orchidaceae and development of molecular markers for phylogenetic applications," PLOS ONE, vol. 9, no. 6, Article ID e99016, 2014.

[47] J. D. Palmer and W. F. Thompson, "Chloroplast DNA rearrangements are more frequent when a large inverted repeat sequence is lost," Cell, vol. 29, no. 2, pp. 537-550, 1982.

[48] S. H. Strauss, J. D. Palmer, G. T. Howe, and A. H. Doerksen, "Chloroplast genomes of two conifers lack a large inverted repeat and are extensively rearranged.", Proceedings of the National Acadamy of Sciences of the United States of America, vol. 85, no. 11, pp. 3898-3902, 1988.

[49] T. W. Chumley, J. D. Palmer, J. P. Mower et al., "The complete chloroplast genome sequence of Pelargonium $\times$ hortorum: Organization and evolution of the largest and most highly rearranged chloroplast genome of land plants," Molecular Biology and Evolution, vol. 23, no. 11, pp. 2175-2190, 2006.

[50] F. Delsuc, H. Brinkmann, and H. Philippe, "Phylogenomics and the reconstruction of the tree of life," Nature Reviews Genetics, vol. 6, no. 5, pp. 361-375, 2005.

[51] C. X. Chan and M. A. Ragan, "Next-generation phylogenomics," Biology Direct, vol. 8, no. 1, article 3, 2013.

[52] V. V. Goremykin, K. I. Hirsch-Ernst, S. Wölfl, and F. H. Hellwig, "Analysis of the Amborella trichopoda chloroplast genome sequence suggests that Amborella is not a basal angiosperm," Molecular Biology and Evolution, vol. 20, no. 9, pp. 1499-1505, 2003. 


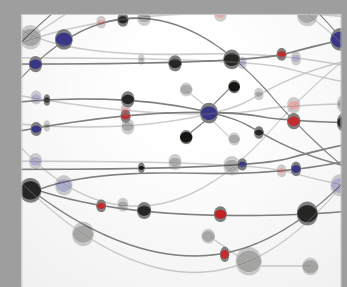

The Scientific World Journal
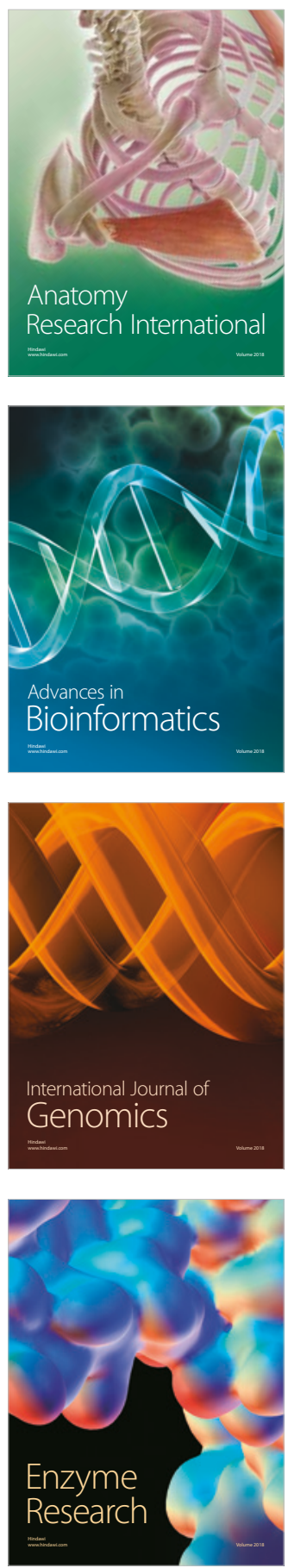
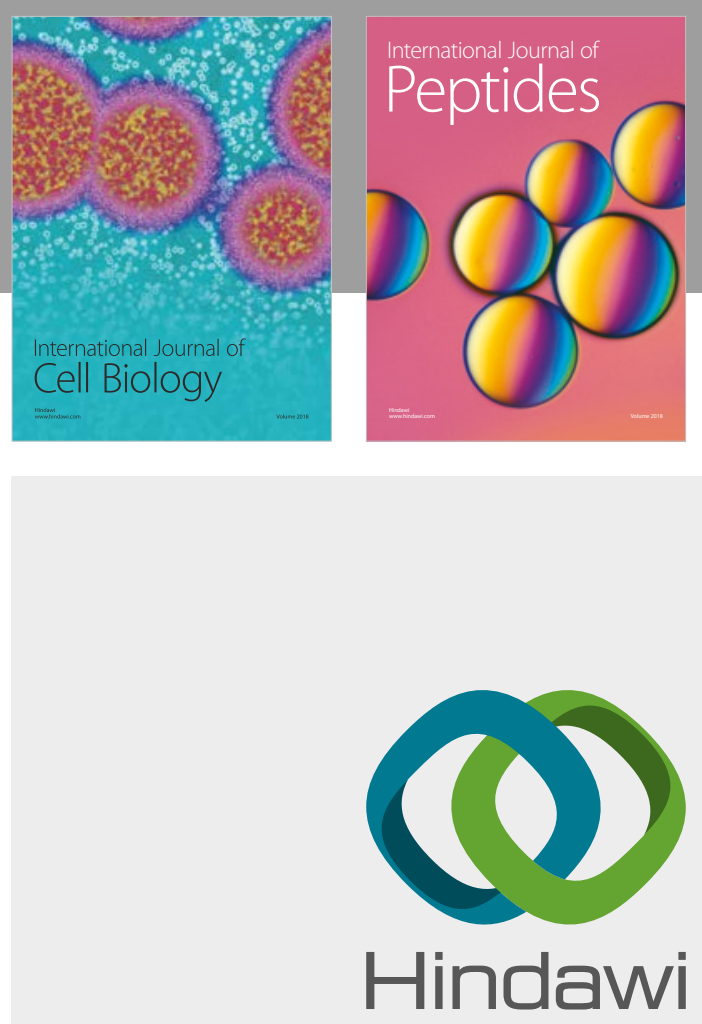

Submit your manuscripts at

www.hindawi.com
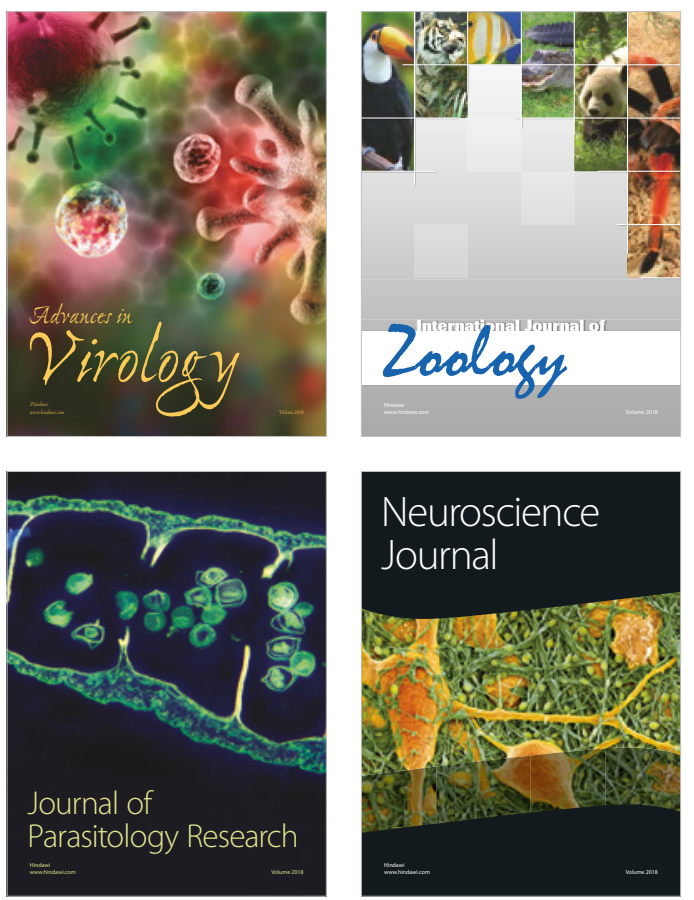
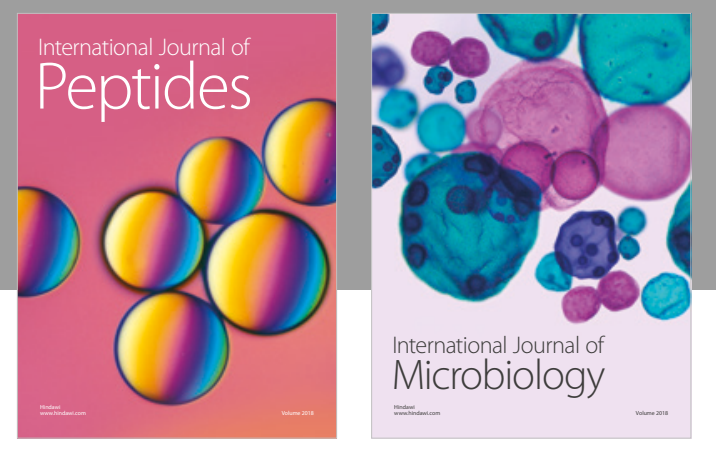

nternational Journal of Microbiology
Journal of
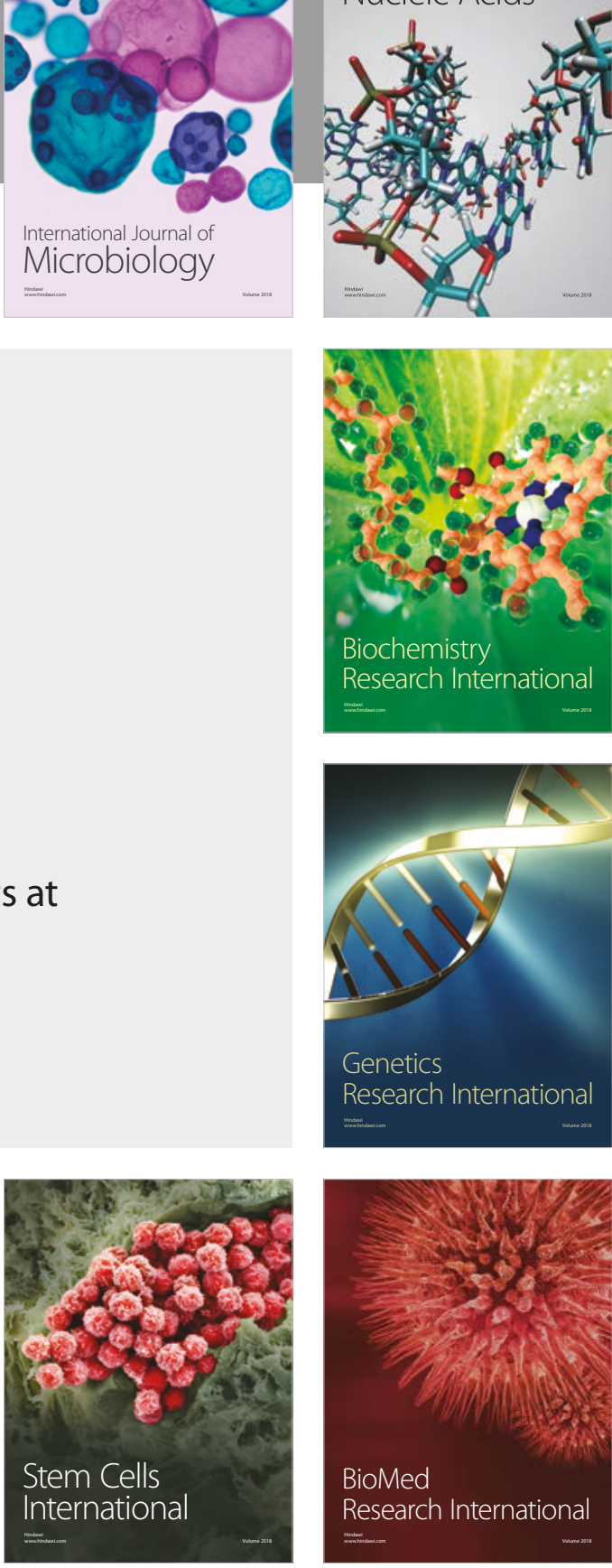
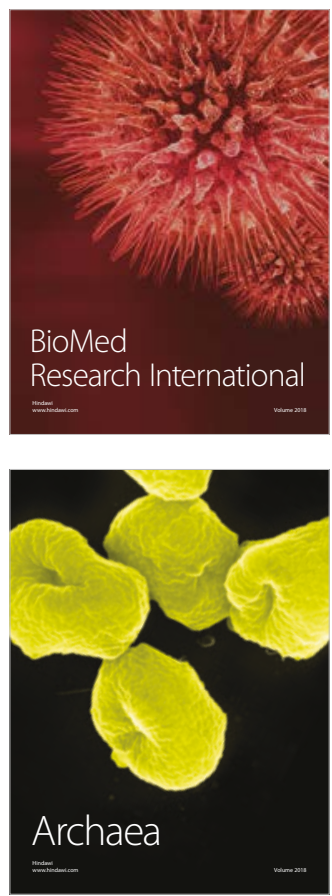